EUROPEAN JOURNAL OF PURE AND APPLIED MATHEMATICS

Vol. 12, No. 2, 2019, 544-552

ISSN 1307-5543 - www.ejpam.com

Published by New York Business Global

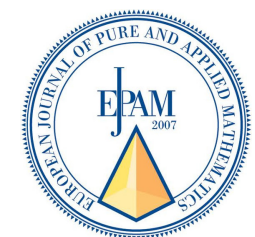

\title{
Weakly Prime and Weakly primary ideals in gamma seminearrings
}

\author{
Waheed Ahmad Khan ${ }^{1}$, Abdelghani Taouti ${ }^{2, *}$, Seema Karkain ${ }^{2}$, \\ Azar Salami ${ }^{2}$, Waqar Arif ${ }^{1}$ \\ 1 Department of Mathematics, University of Education, Attock Campus, Pakistan \\ 2 ETS-Maths and NS Engineering division HCT, University City, P. O. Box: 7947 \\ United Arab Emirates
}

\begin{abstract}
We introduce and discuss the weakly prime and weakly primary ideals of a gamma seminearrings with illustrative examples. We also present few of characterizations of these ideals.
\end{abstract}

2010 Mathematics Subject Classifications: 16Y30, 16Y60

Key Words and Phrases: Gamma seminearrrings, prime ideals, primary ideals.

\section{Introduction and Preliminaries}

The concept of seminearring was introduced by W. G. van Hoorn et al. in [1]. Seminearfields have been introduced in [5]. As a generalization of seminearrings that is $\Gamma$ seminear-rings were introduced in [2]. Subsequently, prime and semiprime ideals in gamma seminearrings have been explored in [3]. In a sequel, we introduce the notion of weakly prime and weakly primary ideals $\Gamma$-seminearring and few of their characterizations. We recall some useful concepts for the sake of completeness. A nonempty set $R$ with two binary operations " + "(addition) and "." (multiplication) is called a seminearring if it satisfies $(i)(R,+)$ and $(R,$.$) are semigroups; (i i)(x+y) . z=x . z+y . z$ for all $x, y, z \in R$. In 2005, Krishna \& Chatterjee [4], introduced the condition of minimality of generalized linear sequential machines using the theory of near-semirings. Near-semirings have proven to be useful in studying automata and formal languages. Following [3], $\Gamma$-seminearring is a triple $(R,+, \Gamma)$ where, $(i) \Gamma$ is a non-empty set of binary operators on $R$ such that for each $\alpha \in \Gamma,(R,+,$.$) is a seminearring, (i i) x \alpha(y \beta z)=(x \alpha y) \beta z$ for all $x, y, z \in R$ and $\alpha, \beta \in$ $\Gamma$. Similarly, let $R$ be a $\Gamma$-seminearring, a subsemigroup $A$ of $(R,+)$ is called a left (resp., right) ideal of $R$ if $R \Gamma A \subseteq A$ (resp., $A \Gamma R \subseteq A$ ). A left and right ideal is called an ideal. Let

\footnotetext{
* Corresponding author.

DOI: https://doi.org/10.29020/nybg.ejpam.v12i2.3397

Email addresses: sirwak2003@ yahoo.com (W. A. Khan), ganitaouti@yahoo.com.au (A. Taouti), skarkain@hct.ac.ae (S. Karkain), asalami@hct.ac.ae (A. Salami), waqarvicky6699@gmail.com (W. Arif) 
$R$ be a $\Gamma$-seminearring and $I, J \subseteq R$. We denote it by $I \Gamma J=\{a \alpha b \mid a, b \in R$ and $\alpha \in \Gamma\}$. A mapping $f: R \rightarrow R^{\prime}$ between two gamma seminearrings is called a $\Gamma$-seminearring homomorphism ( $\Gamma$-homomorphism), if $f(x+y)=f(x)+f(y)$ and $f(x \gamma y)=f(x) \gamma f(y)$ for all $x, y \in R$ and $\gamma \in \Gamma$. Let $R$ and $R^{\prime}$ be a $\Gamma$-seminearrings and $\mathrm{f}: R \rightarrow R^{\prime}$ be a $\Gamma$-seminearring homomorphism. Then, (i) $f\left(I_{1} \Gamma I_{2}\right)=f\left(I_{1}\right) \Gamma f\left(I_{2}\right)$ for all $I_{1}, I_{2} \in R$, (ii) $f^{-1}\left(J_{1}\right) \Gamma f^{-1}\left(J_{2}\right)=f^{-1}\left(J_{1} \Gamma J_{2}\right)$ for all $J_{1}, J_{2} \in R^{\prime}$.

\section{Weakly prime and weakly primary ideals in $\Gamma$-seminearrings}

In this section we introduce the notion of weakly prime and weakly primary ideals in $\Gamma$-seminearrings. By an ideal we mean two-sided ideal unless otherwise stated. We begin with the following definition.

Definition 1. Let $R$ be a $\Gamma$-seminearring. A proper ideal $P$ of $R$ is called weakly prime if for ideals $I$ and $J, 0 \neq I \Gamma J \subseteq P$ implies $I \subseteq P$ or $J \subseteq P$.

Proposition 1. Let $P$ be a proper ideal of a $\Gamma$-seminearring $R$. The following statements are equivalent.

(i) $P$ is weakly prime.

(ii) For ideals $I$ and $J$ of $R, 0 \neq(I \Gamma J) \subseteq P$ implies $I \subseteq P$ or $J \subseteq P$.

(iii) For elements $i$ and $j$ in $R, i \notin P$ and $j \notin P$ implies $0 \neq(i) \Gamma(j) \nsubseteq P$.

Proof. Following definition1, clearly $(i)$ and $(i i)$ are equivalent. Now, $(i) \Longrightarrow($ iii $)$ Let $P$ be a weakly prime, $i \notin P$ and $j \notin P$. Assume $0 \neq(i) \Gamma(j) \subseteq P \Rightarrow(i) \subseteq P$ or $(j) \subseteq P$. Hence, $i \in P$ or $j \in P$, a contradiction. Thus, $0 \neq(i) \Gamma(j) \nsubseteq P$. (iii) $\Longrightarrow(i)$ Assume that $I \nsubseteq P$ and $J \nsubseteq P$. Then there exists $i \in I \backslash P$ and $j \in J \backslash P$. Hence, $0 \neq(i) \Gamma(j) \subseteq 0 \neq I \Gamma J$ but $0 \neq(i) \Gamma(j) \nsubseteq P$ by $(i i i)$. Thus, $0 \neq I \Gamma J \nsubseteq P$.

Example 1. Let $R=\{0,1, e, a, b, c\}$ be a $\Gamma$-seminearring with $\Gamma=\{\alpha, 1\}$.

\begin{tabular}{|c|c|c|c|c|c|c|}
\hline+ & 0 & 1 & $e$ & $a$ & $b$ & $c$ \\
\hline 0 & 0 & 1 & $e$ & $a$ & $a$ & $c$ \\
\hline 1 & 1 & 1 & 1 & 1 & 1 & 1 \\
\hline$e$ & $e$ & 1 & $e$ & 1 & 1 & $e$ \\
\hline$a$ & $a$ & 1 & 1 & $a$ & $a$ & $a$ \\
\hline$b$ & $a$ & 1 & 1 & $a$ & $a$ & $a$ \\
\hline$c$ & $c$ & 1 & $e$ & $a$ & $a$ & $c$ \\
\hline
\end{tabular}




\begin{tabular}{|c|c|c|c|c|c|c|}
\hline$\alpha$ & 0 & 1 & $e$ & $a$ & $b$ & $c$ \\
\hline 0 & 0 & 0 & 0 & 0 & 0 & 0 \\
\hline 1 & 0 & 1 & $e$ & $a$ & $b$ & $c$ \\
\hline$e$ & 0 & $e$ & $e$ & 0 & $c$ & $c$ \\
\hline$a$ & 0 & $a$ & 0 & $a$ & $a$ & 0 \\
\hline$b$ & 0 & $b$ & 0 & $a$ & $a$ & 0 \\
\hline$c$ & 0 & $c$ & 0 & 0 & 0 & 0 \\
\hline
\end{tabular}

$P=\{0, a, b\}$ of a seminearring $R$ is a weakly prime ideal but not a prime ideal, since $c \alpha c=0$ and $c \notin P$. On the other hand, consider a prime ideal $Q=\{0, e, c\}$ of $R$. It is easy to show that $Q$ is a weakly prime ideal. For this, let $I$ and $J$ are ideals of $R$, where $I=\{0, e, c\}$ and $J=\{0, a, b\}$. Then, $0 \neq I \Gamma J \subseteq Q \Longrightarrow I \subseteq Q \Longrightarrow Q$ is a weakly prime ideal. Hence every prime ideal of a gamma seminearring is a weakly prime ideal.

Example 2. Let $R=\{0,1, e, a, b, c\}$ be a $\Gamma$-seminearring with $\Gamma=\{\alpha, 1\}$ as defined in example1. Let $S=\{0,1, e, a, c\} \subseteq R$ be a $\Gamma$-sub-seminearring of $R$ with $\Gamma=\{1$, $\alpha\}$.Clearly, in $S$ the ideals $I=\{0, a\}$ and $J=\{0, c\}$ are weakly prime ideals but not prime.

Proposition 2. Let $P$ be a proper ideal of a $\Gamma$-seminearring $R$ and $\{0 \neq a \alpha r \beta b: r \in R$, $\alpha, \beta \in \Gamma\} \subseteq P$ if and only if $a \in P$ or $b \in P$, then $P$ is a weakly prime ideal.

Proof. Let $I$ and $J$ are ideals of $R$ with $0 \neq I \Gamma J \subseteq P$. Let $I \nsubseteq P$, and for $a \in I \backslash P$, $b \in J$, we have $\{0 \neq a \alpha r \beta b: r \in R, \alpha, \beta \in \Gamma\} \subseteq I \Gamma J \neq 0 \subseteq P$. Since $a \notin P$ and $b \in P \Rightarrow$ $J \subseteq P$. Hence, $P$ is a weakly prime ideal.

Proposition 3. Intersection of finite numbers of weakly prime ideals of a $\Gamma$-seminearring $R$ which are totally ordered by inclusion is a weakly prime ideal.

Proof. Let $\left\{P_{\alpha}\right\}_{\alpha \in \Lambda}$ be the family of weakly prime ideals which are totally ordered by inclusion. Suppose $I$ and $J$ be ideals of $R$. If $0 \neq I \Gamma J \subseteq \cap_{\alpha \in \Lambda} P_{\alpha}$, then $0 \neq I \Gamma J \subseteq P_{\alpha}$, for all $\alpha \in \Lambda$. Suppose that there exists $\alpha \in \Lambda$ such that $I \nsubseteq P_{\alpha}$. Then, $J \subseteq P_{\alpha}$ and hence $J \subseteq P_{\beta}$ for all $\beta \geq \alpha$. We assume that there exist $\gamma<\alpha$ such that $J \subseteq P_{\gamma}$. Then, $I \subseteq P_{\gamma}$ and hence $I \subseteq P_{\alpha}$, which is impossible. Hence, $J \subseteq P_{\beta}$ for any $\beta \in \Lambda$. Thus, $\cap_{\alpha \in \Lambda} P_{\alpha}$ is a weakly prime ideal of a $\Gamma$-seminearring $R$.

Below we provide an illustrative example.

Example 3. Let $S=\{0,1, e, a, c\}$ with $\Gamma=\{1, \alpha\}$ be a $\Gamma$-seminearring defined in the tables given below.

\begin{tabular}{|c|c|c|c|c|c|}
\hline+ & 0 & 1 & $e$ & $a$ & $c$ \\
\hline 0 & 0 & 1 & $e$ & $a$ & $c$ \\
\hline 1 & 1 & 1 & 1 & 1 & 1 \\
\hline$e$ & $e$ & 1 & $e$ & 1 & $e$ \\
\hline$a$ & $a$ & 1 & 1 & $a$ & $a$ \\
\hline$c$ & $c$ & 1 & $e$ & $a$ & $c$ \\
\hline
\end{tabular}

\begin{tabular}{|c|c|c|c|c|c|}
\hline$\alpha$ & 0 & 1 & $e$ & $a$ & $c$ \\
\hline 0 & 0 & 0 & 0 & 0 & 0 \\
\hline 1 & 0 & 1 & $e$ & $a$ & $c$ \\
\hline$e$ & 0 & $e$ & $e$ & 0 & $c$ \\
\hline$a$ & 0 & $a$ & 0 & $a$ & 0 \\
\hline$c$ & 0 & $c$ & 0 & 0 & 0 \\
\hline
\end{tabular}


Consider $P_{1}=\{0, c\}$ and $P_{2}=\{0, e, c\}$ are the weakly prime ideals of $R$ and are totally ordered by inclusion as well. Since, $P_{1} \cap P_{2}=P_{1}$, which is a weakly prime ideal of $R$. Hence, $\cap_{\alpha \in A} P_{\alpha}$ is a weakly prime ideal.

Proposition 4. Let $I$ be an ideal of a $\Gamma$-seminearring $R$ with $R+I \subseteq I$ and $I+R \subseteq I$. Let $P$ be a proper ideal of $R$ containing $I$ and $\psi: R \rightarrow R / I$ be the canonical epimorphism. Then, $P$ is a weakly prime ideal if and only if $\psi(P)$ is a weakly prime.

Proof. Let $P$ be a weakly prime ideal of $R$. Suppose $J_{1}$ and $J_{2}$ are ideals in $R / I$ such that $0 \neq J_{1} \Gamma J_{2} \subseteq \psi(P)$. Assume that $\psi^{-1}\left(J_{1}\right)=I_{1}$ and $\psi^{-1}\left(J_{2}\right)=I_{2}$. Then, $0 \neq I_{1} \Gamma I_{2}=0 \neq \psi^{-1}\left(J_{1}\right) \Gamma \psi^{-1}\left(J_{2}\right) \subseteq 0 \neq \psi^{-1}\left(J_{1} \Gamma J_{2}\right) \subseteq 0 \neq \psi^{-1}(\pi(P))=P$. Since $P$ is a weakly prime ideal, it implies $I_{1} \subseteq P$ or $I_{2} \subseteq P$. Hence, $J_{1}=\psi\left(\psi^{-1}\left(J_{1}\right)\right)=\psi\left(I_{1}\right) \subseteq \psi(P)$ or $J_{2}=\psi\left(\psi^{-1}\left(J_{2}\right)\right)=\psi\left(J_{2}\right) \subseteq \psi(P)$. Hence, $\psi(P)$ is a weakly prime. Conversely, suppose $\psi(P)$ be a weakly prime ideal and let $I_{1}, I_{2}$ are ideals of $R$ such that $0 \neq I_{1} \Gamma I_{2} \subseteq P$. Then, $0 \neq \psi\left(I_{1}\right) \Gamma \psi\left(I_{2}\right)=0 \neq \psi\left(I_{1} \Gamma I_{2}\right) \subseteq \psi(P)$. Since $\psi(P)$ is a weakly prime ideal, it implies that $\psi\left(I_{1}\right) \subseteq \psi(P)$ or $\psi\left(I_{2}\right) \subseteq \psi(P)$. Thus, $I_{1} \subseteq P$ or $I_{2} \subseteq P$, and hence $P$ is a weakly prime ideal of a $\Gamma$-seminearring $R$.

Definition 2. Let $R$ be a $\Gamma$-seminearring and $M$ be a non-empty subset of $R$. We call $M$ an $m$-system if for $a, b \in M$, there exist $a_{1} \in(a), b_{1} \in(b)$ and $\alpha \in \Gamma$ such that $0 \neq a_{1} \alpha b_{1} \in M$.

Proposition 5. Let $P$ be a proper ideal of a $\Gamma$-seminearring $R$. Then, $P$ is a weakly prime ideal if and only if $R \backslash P$ is m-system.

Proof. Let $P$ be a weakly prime ideal of a $\Gamma$-seminearring $R$. Consider $a, b \in R \backslash P$ and $0 \neq(a) \Gamma(b) \nsubseteq P$. Let $a_{1} \in(a), b_{1} \in(b)$ and $\alpha \in \Gamma$ such that $0 \neq a_{1} \alpha b_{1} \notin P$, i.e., $a_{1} \alpha b_{1} \in R \backslash P$. Thus, $R \backslash P$ is an $m$-system. Conversely, suppose $R \backslash P$ is an $m$-system and let $a, b \in R \backslash P$. Then, there exist $a_{1} \in(a), b_{1} \in(b)$ and $\alpha \in \Gamma$ such that $a_{1} \alpha b_{1} \in R \backslash P$. Thus, $0 \neq(a) \Gamma(b) \nsubseteq P$ and hence $P$ is a weakly prime ideal of a $\Gamma$-seminearring $R$.

Definition 3. $A$ subset $A$ of $a \Gamma$-seminearring $R$ is a subtractive, if $a \in A$ and $a+b \in A$ implies $b \in A$.

Proposition 6. Let $R$ be a $\Gamma$-seminearring whose all ideals are subtractive, and let $P$ be a proper ideal of $R$. Then, $P$ is a weakly prime if and only if for any ideals $I, J$ of $R$, $P \subset I$ and $P \subset J$ implies $0 \neq I \Gamma J \nsubseteq P$.

Proof. Suppose for any ideals $I, J$ of $R, P \subset I$ and $P \subset J$ implies $0 \neq I \Gamma J \nsubseteq P$. Let us suppose that $I \nsubseteq P$ and $J \nsubseteq P$. Then there exist $i \in I \backslash P$ and $j \in J \backslash P$ and hence $P \subset P+(i)$. By hypothesis, $0 \neq(P+(i)) \Gamma(P+(j)) \nsubseteq \nsubseteq P$ and so there exist $i^{\prime} \in(i)$, $j^{\prime} \in(j), p, p^{\prime} \in P$ and $\alpha \in \Gamma$ such that $0 \neq\left(p+i^{\prime}\right) \alpha\left(p^{\prime}+j^{\prime}\right) \notin P$. Since, $0 \neq p \alpha\left(p^{\prime}+j^{\prime}\right) \in P$, $0 \neq i^{\prime} \alpha\left(p^{\prime}+j^{\prime}\right) \notin P$ and $P$ is an ideal, then $i^{\prime} \notin P$ and $p^{\prime}+j^{\prime} \notin P$. Thus, $i^{\prime} \notin P$ and $j^{\prime} \notin P$ because $P$ is subtractive. It implies $0 \neq\left(i^{\prime}\right) \Gamma\left(j^{\prime}\right) \nsubseteq P$. But $0 \neq\left(i^{\prime}\right) \Gamma\left(j^{\prime}\right) \subseteq 0 \neq$ $I \Gamma J \Rightarrow 0 \neq I \Gamma J \nsubseteq P$. Hence, $P$ is a weakly prime ideal. The converse is obvious by the definition of a weakly prime ideal of a $\Gamma$-seminearring. 
Theorem 1. Let $M$ be an $m$-system of a $\Gamma$-seminearring $R$ whose each ideal is a subtractive. Let $I$ be an ideal with $I \cap M=\emptyset$. Then, there exists a weakly prime ideal $P$ such that $I \subseteq P$ and $P \cap M=\emptyset$.

Proof. Let $\Im=\{J: J$ is an ideal of $R, I \subseteq J$ and $J \cap M \neq \emptyset\}$. Then, $\Im \neq \emptyset$ and let $\left\{J_{\alpha}\right\}_{\alpha \in A}$ be a chain in $I$ which is ordered under set inclusion. Then, $I \subseteq \cap_{\alpha \in \Lambda} J_{\alpha}$ and $\left(\cup_{\alpha \in \Lambda} J_{\alpha}\right) \cap M=\cup_{\alpha \in \Lambda}\left(J_{\alpha} \cap M\right) \neq \emptyset$. Thus, $\cup_{\alpha \in \Lambda} J_{\alpha} \in I$. By Zorn's Lemma, $\Im$ has a maximal element say $P$. We also claim that $P$ is a weakly prime ideal. If $P \subset K_{1}$ and $P \subset K_{2}$, then there exist $k_{1} \in K_{1} \cap M, k_{2} \in K_{2} \cap M$ and $\alpha \in \Gamma$ such that $0 \neq\left(k_{1}\right) \alpha\left(k_{2}\right) \subseteq$ $0 \neq K_{1} \Gamma K_{2}$ and there exist $k_{1}^{\prime} \in\left(k_{1}\right)$ and $k_{2}^{\prime} \in\left(k_{2}\right)$ such that $0 \neq k_{1}^{\prime} \alpha k_{2}^{\prime} \in M$. Thus, $0 \neq$ $k_{1}^{\prime} \alpha k_{2}^{\prime} \in 0 \neq K_{1} \Gamma K_{2} \cap M$. Since $P \cap M=\emptyset,\left(K_{1} \Gamma K_{2}\right) \nsubseteq P$. Hence, $P$ is a weakly prime ideal.

Now we present few results about such a $\Gamma$-seminearring $R$ in which each ideal is weakly prime.

Proposition 7. Every ideal of a $\Gamma$-seminearring $R$ is a weakly prime if and only if for any ideals $I, J, K$ of $R, I \Gamma J=I, I \Gamma J=J, I \Gamma J=K$ where $K$ is the ideal contained in both $I$ and $J$, or $I \Gamma J=0$.

Proof. Suppose that every ideal of $R$ is a weakly prime. Let $I, J$ are ideals of a $\Gamma$-seminearring $R$. If $I \Gamma J \neq R$, then $I \Gamma J$ is a weakly prime. If $0 \neq I \Gamma J \subseteq I \Gamma J$, then we have $I \subseteq I \Gamma J$ or $J \subseteq I \Gamma J$ i.e., $I=I \Gamma J$ or $J=I \Gamma J$. If $I \Gamma J=K$ then clearly $K=I \cap J$ is a weakly prime ideal then by proposition3, $K \subset I$ and $K \subset J$. Finally, if $I \Gamma J=R$, then we have $I=J=R$ and hence $R \Gamma R=R$.

Conversely, let $L$ be any proper ideal of $R$ and suppose that $0 \neq I \Gamma J \subseteq L$ for ideals $I$ and $J$ of $R$. Then, we have either $I=I \Gamma J \subseteq L$ or $J=I \Gamma J \subseteq L$. And if $K=I \Gamma J \subseteq L$, where $K \subset I \cap J$ and hence $K \cap I \subseteq I$ and $K \cap J \subseteq L$.

Example 4. Refer to the $\Gamma$-seminearring $S$ defined by tables in example3. Clearly, $S$ has four ideals, $I=\{0, a\}, J=\{0, c\}, K=\{0, e, c\}$ and $L=\{0, a, c\}$. Now, $I \Gamma J=\{0\}$, $I \Gamma K=\{0\}, I \Gamma L=I, J \Gamma I=\{0\}, J \Gamma K=\{0\}, J \Gamma L=\{0\}, K \Gamma I=\{0\}, K \Gamma J=J$, $K \Gamma L=J$ where $J \subset K$ and $J \subset L, L \Gamma I=I, L \Gamma J=\{0\}, L \Gamma K=\{0\}$. Hence, we can check easily that every ideal of $S$ is a weakly prime ideal.

Corollary 1. Let $R$ be a $\Gamma$-seminearring in which every ideal of $R$ is a weakly prime. Then for any ideal $I$ of $R$, either $I \Gamma I=I^{2}=I$ or $I \Gamma I=I^{2}=0$.

Example 5. Refer to example4, since $I=\{0, a\}$ be the weakly prime ideal of $S$ and hence $I \Gamma I=I^{2}=I$. Also, for another weakly prime ideal $J=\{0, c\}$ of $S$ we have $J \Gamma J=J^{2}=0$.

In the above example the ideal $K=\{0, e, c\}$ we have $K \Gamma K=K^{2}=\{0, e\}$ which is a subset of $\Gamma$-seminearring but not an ideal. And for the ideal $L=\{0, a, c\}$ we have $L \Gamma L=L^{2}=\{0, a\}$ which is a weakly prime ideal of $S$.)

Proposition 8. Suppose that every ideal of a $\Gamma$-seminearring $R$ is a weakly prime. If $M_{1}$ and $M_{2}$ are two maximal ideals of $R$ then $M_{1} \Gamma M_{2}=0$ or $M_{1} \Gamma M_{2}=N=M_{1} \cap M_{2}$. 
Proof. Suppose every ideal of a $\Gamma$-seminearring $R$ is a weakly prime ideal. Let $M_{1}$ and $M_{2}$ be the two distinct maximal ideals. Since, $M_{1} \cap M_{2}$ is a weakly prime and hence $M_{1} \Gamma M_{2} \subseteq M_{1} \cap M_{2}$, we must have $M_{1} \Gamma M_{2}=0$ and similarly $M_{2} \Gamma M_{1}=0$, or $M_{2} \Gamma M_{1}=N$, being every ideal a weakly prime ideal of $R$, the result follows from proposition3.

Example 6. Refer to the $\Gamma$-seminearring $S$ defined in tables of an example3. Let $I=\{0$, $a, c\}$ and $J=\{0, e, c\}$ be the two maximal ideals of $S$. Clearly $I \Gamma J=0$ and $J \Gamma I=\{0$, $c\}=I \cap J=\{0, c\}$.

Corollary 2. Let every ideal of a $\Gamma$-seminear-ring $R$ is a weakly prime. Then, every nonzero ideal of $R / N(R)$ is prime.

Corollary 3. Suppose that every ideal of a $\Gamma$-seminear-ring $R$ is a weakly prime. Then $(N(R)) \Gamma(N(R))=0$ and every prime ideal $P(R)$ contains $N(R)$. There are three possibilities.

(a) $N(R)=R$.

(b) $N(R)=P(R)$ is the smallest prime ideal and all other prime ideals are idempotent and are linearly ordered. If $N(R) \neq 0$, then it is the only non-idempotent prime ideal. (c) $N(R)=P(R)$ is not a prime ideal. And in such case there exist two nonzero minimal prime ideals $J_{1}$ and $J_{2}$ with $N(R)=J_{1} \cap J_{2}$ and $J_{1} \Gamma J_{2}=\{0\}$ or $(d), J_{2} \Gamma J_{1}=\{0\}$ or $(c)$. All other ideals containing $N(R)$ also contain $J_{1}+J_{2}$ and they are linearly ordered.

We elaborate the above proposition in the below example.

Example 7. Refer to the $\Gamma$-seminearring $S$ defined in tables of an example3. In $S, N(S)=$ $\{0, c\}$ and we have $(N(S))^{2}=(N(R)) \Gamma(N(R))=\{0, c\}^{2}=\{0\}$. As, $N(S)=P(S)$ is not a prime ideal and possibility $(c)$ of the above corollary 3 is valid for this i.e., there exist two nonzero minimal prime ideals $J_{1}$ and $J_{2}$ with $N(R)=J_{1} \cap J_{2}$ and $J_{1} \Gamma J_{2}=\{0\}$ and $J_{2} \Gamma J_{1}=(c)=\{0, c\}$. All other ideals containing $N(R)$ also contain $J_{1}+J_{2}$ and are linearly ordered. Let $J_{1}=\{0, a, c\}$ and $J_{2}=\{0, e, c\}$ be the minimal prime ideals of $S$. We have $N(S)=\{0, c\}=J_{1} \cap J_{2}$ and $J_{1} J_{2}=J_{2} J_{1}=0$. Beside these two ideals another ideal of $S$ is $S$ itself and clearly it contains $N(S)$ and also $J_{1}+J_{2}$ where $J_{1}+J_{2}=S$.

Example 8. Let $T=\{0, a, b\}$ be a right seminearring under the operations defined in given below tables.

\begin{tabular}{|c|c|c|c|}
\hline+ & 0 & $a$ & $b$ \\
\hline 0 & 0 & $a$ & $b$ \\
\hline$a$ & $a$ & $a$ & $a$ \\
\hline$b$ & $b$ & $b$ & $b$ \\
\hline
\end{tabular}

\begin{tabular}{|l|l|l|l|}
\hline. & 0 & $a$ & $b$ \\
\hline 0 & 0 & 0 & 0 \\
\hline$a$ & 0 & $a$ & $a$ \\
\hline$b$ & 0 & $a$ & $b$ \\
\hline
\end{tabular}

Here $N(T)=P(T)=\{0\}$ and it is the smallest prime ideal. Possibility (b) of above corollary3 is valid for this seminearring.

Definition 4. Let $R$ be a $\Gamma$-seminearring under the mapping from $R \times \Gamma \times R$ into $R$, say $f$, and $D$ be the set of all destributive elements of $R$, i.e., $D=\{d \in R \mid d \alpha(a+b)=$ 
$d \alpha a+d \alpha b$ for all $\mathrm{a}, \mathrm{b} \in R$ and $\alpha \in \Gamma\}$. Then $R$ is called distributively generated (in short, d.g.) if the set $D$ is non empty subset of $R$ which $f_{D \times \Gamma \times D}: D \times \Gamma \times D \rightarrow D$ and $(<D,+>)=(R,+)$ where $<D>=\left\{\sum_{i=1}^{m} \alpha_{i} d_{i} \mid m, \alpha_{i} \in N\right.$ and $d_{i} \in D$ for all $\left.i\right\}$. In fact, $<D>=\left\{\sum_{i=1}^{n} d_{i} \mid n \in N\right.$ and $\left.d_{i} \in D\right\}$ where all $d_{i}^{\prime} s$ in $\sum d_{i}$ may not be distinct. In addition, $(<D,+>)=(R,+)$ means that every element in $R$ can be written as a finite sum of desrtributive elements.

Example 9. Refer to the $\Gamma$-seminearring $S$ defined in tables of an example3. Let $D=\{0$, $1\}$, where all elements of $D$ are distributive elements of $R$ i.e., $D=\{d \in R \mid d \alpha(a+b)=$ $d \alpha a+d \alpha b$ for all $a, b \in R$ and $\alpha \in \Gamma\}$. $S$ is called distributively generated because the set $D=\{0,1\}$ is a nonempty subset of $R$ which satisfies $f_{D \times \Gamma \times D}: D \times \Gamma \times D \rightarrow D$ and $(<D>,+)=(R,+)$.

Theorem 2. Let $R$ be a distributively generated $\Gamma$-seminearring.

(1) If $A$ is weakly prime ideal of $R$ and $B$ is a nonempty subset of $R$. Then, $A \Gamma B$ is a weakly prime ideal of $R$.

(2) If $A$ and $B$ are weakly prime ideals of $R$, then $A \Gamma B$ is an ideal of $R$.

Example 10. Refer to the $\Gamma$-seminearring $S$ defined in tables of an example3. Let $A=\{0$, a\} be a weakly prime ideal of $R$ and $B=\{1, e\}$ be a nonempty subset of $R$. Clearly $A \Gamma B=\{0, a\}$ is a weakly prime ideal of $S$. Let $C=\{0, c\}$ be another weakly prime ideal. Also $A \Gamma C=\{0\}$ and it is a minimal prime ideal of $S$.

\section{Weakly primary ideals}

Definition 5. Let $R$ be a $\Gamma$-seminearring. A proper ideal $P$ of $R$ is said to be a weakly primary ideal if $0 \neq p \gamma q \in P$ implies $p \in P$ or $q^{n} \in P$.

\begin{tabular}{|c|c|c|c|c|c|c|}
\hline+ & 0 & 1 & $e$ & $a$ & $b$ & $c$ \\
\hline 0 & 0 & 1 & $e$ & $a$ & $a$ & $c$ \\
\hline 1 & 1 & 1 & 1 & 1 & 1 & 1 \\
\hline$e$ & $e$ & 1 & $e$ & 1 & 1 & $e$ \\
\hline$a$ & $a$ & 1 & 1 & $a$ & $a$ & $a$ \\
\hline$b$ & $a$ & 1 & 1 & $a$ & $a$ & $a$ \\
\hline$c$ & $c$ & 1 & $e$ & $a$ & $a$ & $c$ \\
\hline
\end{tabular}

\begin{tabular}{|c|c|c|c|c|c|c|}
\hline$\alpha$ & 0 & 1 & $e$ & $a$ & $b$ & $c$ \\
\hline 0 & 0 & 0 & 0 & 0 & 0 & 0 \\
\hline 1 & 0 & 1 & $e$ & $a$ & $b$ & $c$ \\
\hline$e$ & 0 & $e$ & $e$ & 0 & $c$ & $c$ \\
\hline$a$ & 0 & $a$ & 0 & $a$ & $a$ & 0 \\
\hline$b$ & 0 & $b$ & 0 & $a$ & $a$ & 0 \\
\hline$c$ & 0 & $c$ & 0 & 0 & 0 & 0 \\
\hline
\end{tabular}


Example 11. Let $R=\{0,1, e, a, b, c\}$ be a $\Gamma$-seminearring with $\Gamma=\{\alpha, 1\}$ defined in example1. Here $I=\{0, a\}, J=\{0, a, c\}$ are weakly primary ideals but not a weakly prime. Clearly, $b \alpha b=a \in I$ but $b^{2}=a \in I$. Similarly, $J$ is also a weakly primary but not a weakly prime ideal. neither prime because in $J$, as $e . b=c \in J$. Clearly, $e, b \notin J$ but $b^{2}=a \in J$.

Proposition 9. Every weakly prime ideal is a weakly primary ideal but converse is not true.

Example 12. Let $R=\{0,1, e, a, b, c\}$ be a $\Gamma$-seminearring with $\Gamma=\{\alpha, 1\}$. In $R$ the ideal $I=\{0, a, b\}$ is weakly prime and also by above proposition it is weakly primary but it is not prime $\mathrm{b} / \mathrm{c} c \alpha c=0$ and $c \notin I$. Another ideal $J=\{0, a\}$ is weakly primary but not weakly prime neither prime $\mathrm{b} / \mathrm{c} b \alpha b=a \in I$. Clearly, $b \notin I$ but $b^{2}=a \in I$.

Proposition 10. Intersection of finite numbers of weakly primary ideals of a $\Gamma$-seminearring $R$ which are totally ordered by inclusion is a weakly primary ideal.

Proof. Let $\left\{P_{\alpha}\right\}_{\alpha \in \Lambda}$ be the family of weakly primary ideals which are totally ordered by inclusion. Suppose $I$ and $J$ be ideals of $R$. If $0 \neq I \Gamma J \subseteq \cap_{\alpha \in \Lambda} P_{\alpha}$, then $0 \neq I \Gamma J \subseteq P_{\alpha}$, for all $\alpha \in \Lambda$. Suppose that there exists $\alpha \in \Lambda$ such that $I \nsubseteq P_{\alpha}$. Then, $J^{n} \subseteq P_{\alpha}$ and hence $J^{n} \subseteq P_{\beta}$ for all $\beta \geq \alpha$. We assume that there exist $\gamma<\alpha$ such that $J^{n} \subseteq P_{\gamma}$. Then, $I \subseteq P_{\gamma}$ and hence $I \subseteq P_{\alpha}$, which is impossible. Hence, $J^{n} \subseteq P_{\beta}$ for any $\beta \in \Lambda$. Thus, $\cap_{\alpha \in \Lambda} P_{\alpha}$ is a weakly primary ideal of a $\Gamma$-seminearring $R$.

Example 13. Let $R=\{0,1, e, a, b, c\}$ be a $\Gamma$-seminearring with $\Gamma=\{\alpha, 1\}$. Here $I=\{0, a\}, J=\{0, a, c\}$ are weakly primary ideals but not weakly prime and $K=\{0, a, b, c\}$ is prime and hence weakly primary because every prime ideal is weakly primary. Clearly, these ideals are totally ordered by inclusion i.e. $I \subseteq J \subseteq K$. Since, $I \cap J \cap K=I=\{0, a\}$, which is also a primary ideal $b / c b . b=a \in I$. Clearly, $b \notin I$ but $b^{2}=a \in I$.

Proposition 11. Every ideal of a $\Gamma$-seminearring $R$ is a weakly primary if and only if for any ideals $I, J, K$ of $R, I \Gamma J=I, I \Gamma J=J, I \Gamma J=K$ where $K$ is the ideal contained in both $I$ and $J$ or either in $I$ or in $J$ i.e. $K \subseteq I, J$ or $K \subseteq I$ or $K \subseteq J$, or $I \Gamma J=0$.

Proof. Suppose that every ideal of $R$ is a weakly prime. Let $I, J$ are ideals of a $\Gamma$-seminearring $R$. If $I \Gamma J \neq R$, then $I \Gamma J$ is a weakly prime. If $0 \neq I \Gamma J \subseteq I \Gamma J$, then we have $I \subseteq I \Gamma J$ or $J^{n} \subseteq I \Gamma J$ i.e., $I=I \Gamma J$ or $J^{n}=I \Gamma J$. If $I \Gamma J=K$ then clearly $K=I \cap J$ is a weakly primary ideal then, $K \subset I$ and $K \subset J^{n}$. Finally, if $I \Gamma J=R$, then we have $I=J=R$ and hence $R \Gamma R=R$.

Conversely, let $L$ be any proper ideal of $R$ and suppose that $0 \neq I \Gamma J \subseteq L$ for ideals $I$ and $J$ of $R$. Then, we have either $I=I \Gamma J \subseteq L$ or $J^{n}=I \Gamma J \subseteq L$. And if $K=I \Gamma J \subseteq L$, where $K \subset I \cap J$ and hence $K \cap I \subseteq I$ and $K \cap J \subseteq L$.

Example 14. Let $R=\{0,1, e, a, b, c\}$ be a $\Gamma$-seminearring with $\Gamma=\{1, \alpha\}$. As $R$ has six different ideals i.e. $I=\{0, a\}, J=\{0, c\}, K=\{0, e, c\}, L=\{0, a, c\}, M=\{0, a, b\}$, and 
$N=\{0, a, b, c\}$. Now, $I \Gamma I=I, I \Gamma J=\{0\}, I \Gamma K=\{0\}, I \Gamma L=I, I \Gamma M=I, I \Gamma N=I$, $J \Gamma I=\{0\}, J \Gamma J=\{0\}, J \Gamma K=\{0\}, J \Gamma L=\{0\}, J \Gamma M=\{0\}, J \Gamma N=\{0\}, K \Gamma I=\{0\}$, $K \Gamma J=J, K \Gamma K=K, K \Gamma L=J, K \Gamma M=J$ where $J \subseteq K, K \Gamma N=J$, where $J \subseteq K$ and $J \subseteq N, L \Gamma I=I, L \Gamma J=\{0\}, L \Gamma K=\{0\}, L \Gamma L=I$, where $I \subseteq L, L \Gamma M=I$ where $I \subseteq L$ and $I \subseteq M, L \Gamma N=I$, where $I \subseteq L$ and $I \subseteq N, M \Gamma I=I, M \Gamma J=\{0\}, M \Gamma K=\{0\}$, $M \Gamma L=I$ where $I \subseteq M$ and $I \subseteq L, M \Gamma M=I$, where $I \subseteq M, M \Gamma N=I$, where $I \subseteq M$ and $I \subseteq N$. Hence, we can easily check that every ideal of $R$ is weakly primary.

Proposition 12. Suppose that every ideal of a $\Gamma$-seminearring $R$ is a weakly primary. If $M_{1}$ and $M_{2}$ are two maximal ideals of $R$ then either $M_{1} \Gamma M_{2}=0$ or $M_{1} \Gamma M_{2}=N=$ $M_{1} \cap M_{2}$.

Example 15. Let $R=\{0,1, e, a, b, c\}$ be a $\Gamma$ - seminearring with $\Gamma=\{1, \alpha\}$. Let $I=$ $\{0, a, b, c\}$ and $J=\{0, e, c\}$ be the two maximal ideals of $R$. Clearly $I \Gamma J=\{0\}$ and $J \Gamma I=\{0, c\}=I \cap J$.

\section{References}

[1] W. G. Van Hoorn, and B. Van Rootselaar, Fundamental notions in the theory of seminearrings, Compositio Math. 18 (1967), 65-78.

[2] Y. B. Jun and K. H. Kim, On structures of gamma-seminear-rings, (submitted)

[3] K. H. Kim, On prime and semiprime ideals in gamma-seminearrings, Sci. Math. Jap. Online, 4, (2001), 885-889.

[4] K. V. Krishna and N. Chatterjee, A necessary condition to test the minimality of generalized linear sequential machines using the theory of near-semirings, Algebra and Discrete Mathematics. 3 (2005), $30-45$.

[5] H. J. Weinert, Seminear-rings, seminearfieds and their semigroup theoretic background, Semigroup Forum 24 (1982), 235-254. 